\title{
On the relationship between mechanical energy rate and heat dissipated rate during fatigue for a C45 steel depending on stress ratio
}

\author{
Rosa De Finis ${ }^{1}$, Davide Palumbo ${ }^{1}$, and Umberto Galietti ${ }^{1}$ \\ ${ }^{1}$ Politecnico di Bari
}

May 14, 2021

\begin{abstract}
This work deals with the analysis in the frequency domain of the temperature signal and mechanical energy rate of C45 steel under two different fatigue stepwise loading series at stress ratios of 0.1 and -1. It was first investigated the energy distribution among the harmonic components of the signals to understand possible variations caused by a different stress ratio. In addition, the second amplitude harmonic ( $\mathrm{SAH}$ ) of heat dissipated and mechanical energy rates have been considered in the analysis and their relationship was investigated. It has been shown as it depends only on the material, hence it is valid whatever the kind of the test is without any assumption on the energy supplied to the material or material hysteresis loop stabilisation. The adopted approach allows the analysis of intrinsic dissipations by means of rapid, full-field and contactless techniques without any specific requirement on loading condition or temperature signal stabilisation.
\end{abstract}

Rosa De Finis

Politecnico di Bari

Via Orabona 4,

Bari, Italy, 70125

rosa.definis@poliba.it]

Professor Y. Hong,

Editor-in-Chief

Fatigue 83 Fracture of Engineering Materials 83 Structures

May 12, 2021

Dear Editor,

I am pleased to submit an original research article entitled "On the relationship between mechanical energy rate and heat dissipated rate during fatigue for a $\mathrm{C} 45$ steel depending on stress ratio" by Rosa De Finis, Davide Palumbo and Umberto Galietti for consideration for publication in the Fatigue \& Fracture of Engineering Materials $\& 3$ Structures. We previously presented a new procedure to analyze temperature data acquired during a rapid fatigue test and to evaluate the endurance limit of different stainless steels and this manuscript builds on our prior study to investigate more in depth, in frequency domain, the thermal signal acquired during a rapid, stepwise fatigue test. In particular, we focus on the second amplitude harmonic (SAH) of thermal signal, a very well-known parameter to estimate the damage under specific test conditions (fully-reversed applied load). We investigate the capability of SAH of temperature signal to represent an 
useful parameter to estimate the heat dissipated energy at different stress ratio conditions $(R=-1$ and $R=0.1)$. In addition, we study the frequency spectrum of the strain energy rate, as well, in order to understand the energy distribution among the harmonics for different stress ratios.

In this manuscript, we show that in presence of the mean stress in the load, the frequency spectrum of the strain energy density rate presents different harmonic components (not just one as occurs at $R=-1$ ) while heat dissipated energy rate spectrum presents different harmonics, so that the SAH is not the only one but it can be used to estimate the heat dissipated energy. In addition, we show that the second amplitude harmonic (SAH) of the heat energy rate dissipated by a unit volume of material is linearly related to the SAH of strain energy density rate. Such a relationship depends only on material and do not depend on loading conditions or damage level. The relationship is also valid, independently on signal stabilisation.

We believe that this manuscript is appropriate for publication by theFatigue 83 Fracture of Engineering Materials 8 Structuresbecause it regards the use of innovative characterization methods associated with fatigue such as the energy-based methods for carrying out the damage monitoring of the C45 steel. Our manuscript can create a paradigm for future studies for the understanding of the relationship between mechanical energy rate and heat dissipated rate during fatigue tests under different loading conditions.

This manuscript has not been published and is not under consideration for publication elsewhere. We have no conflicts of interest to disclose.

Thank you for your consideration.

Sincerely,

Rosa De Finis, $\mathrm{PhD}$

Post doctoral research fellow, Department of Mechanical Mathematics and Management

Politecnico di Bari-Via Orabona 4, 70125-Bari, Italy

Hosted file

Manuscript.pdf available at https://authorea.com/users/176512/articles/521977-on-therelationship-between-mechanical-energy-rate-and-heat-dissipated-rate-during-fatigue-

for-a-c45-steel-depending-on-stress-ratio 

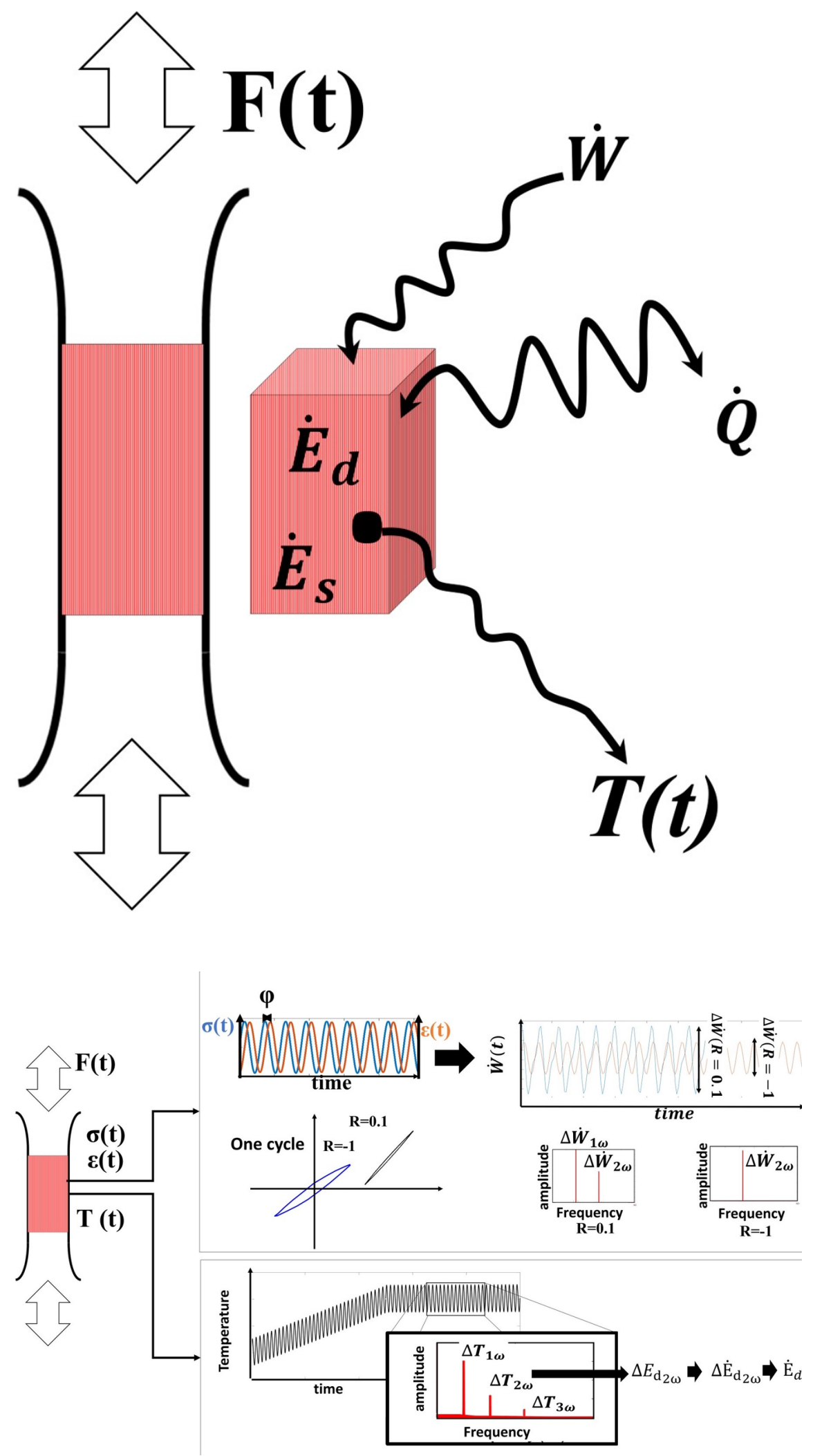


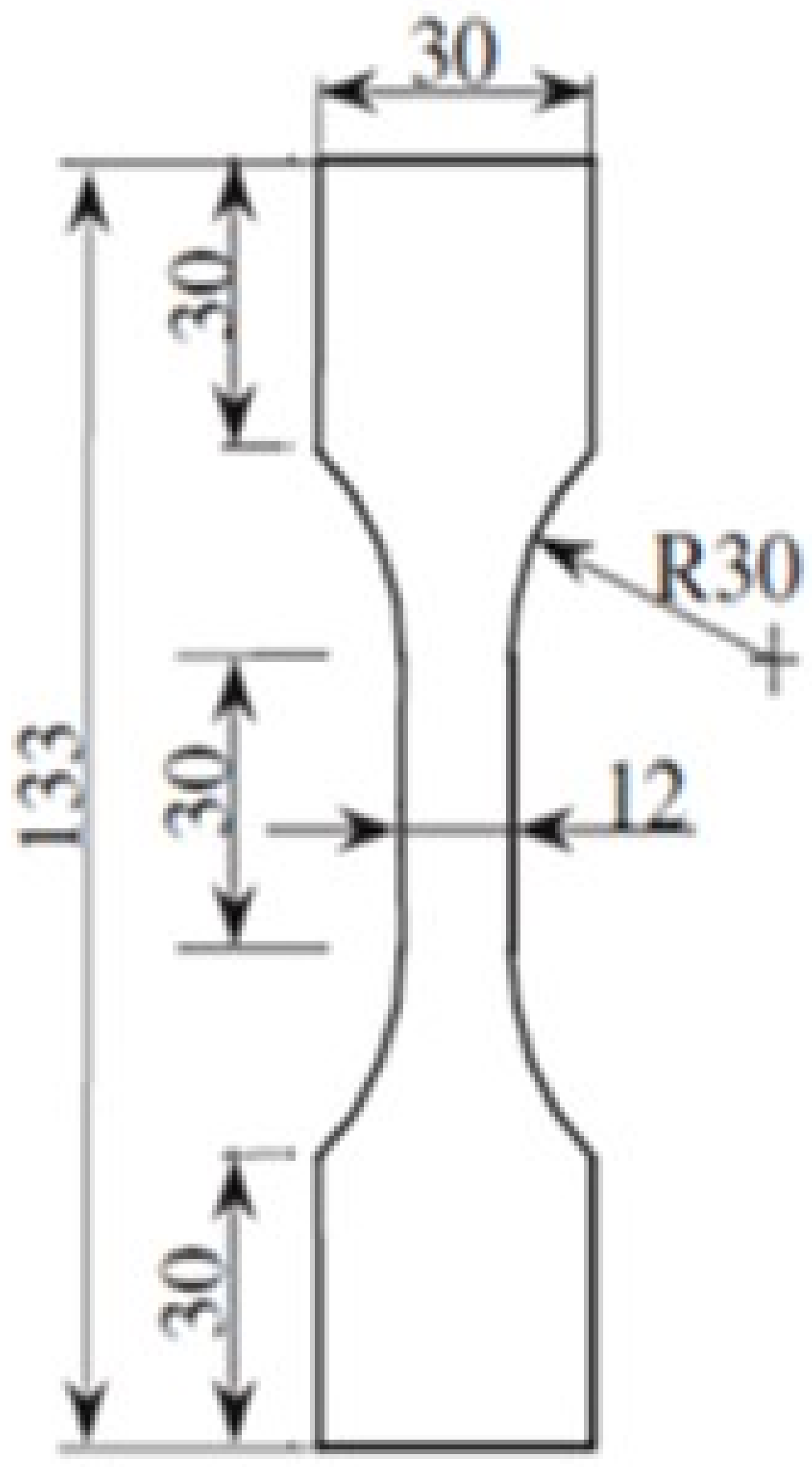



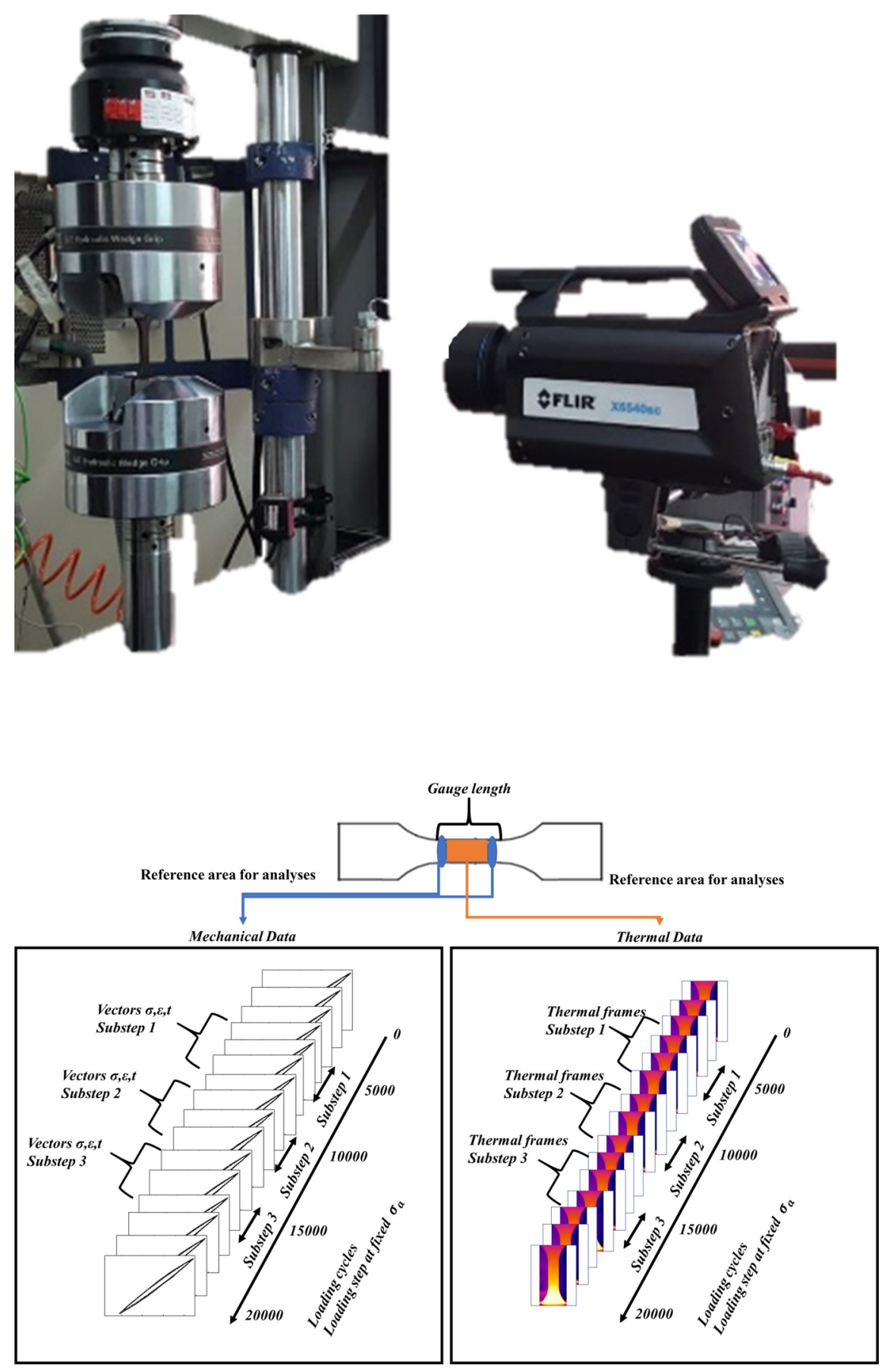

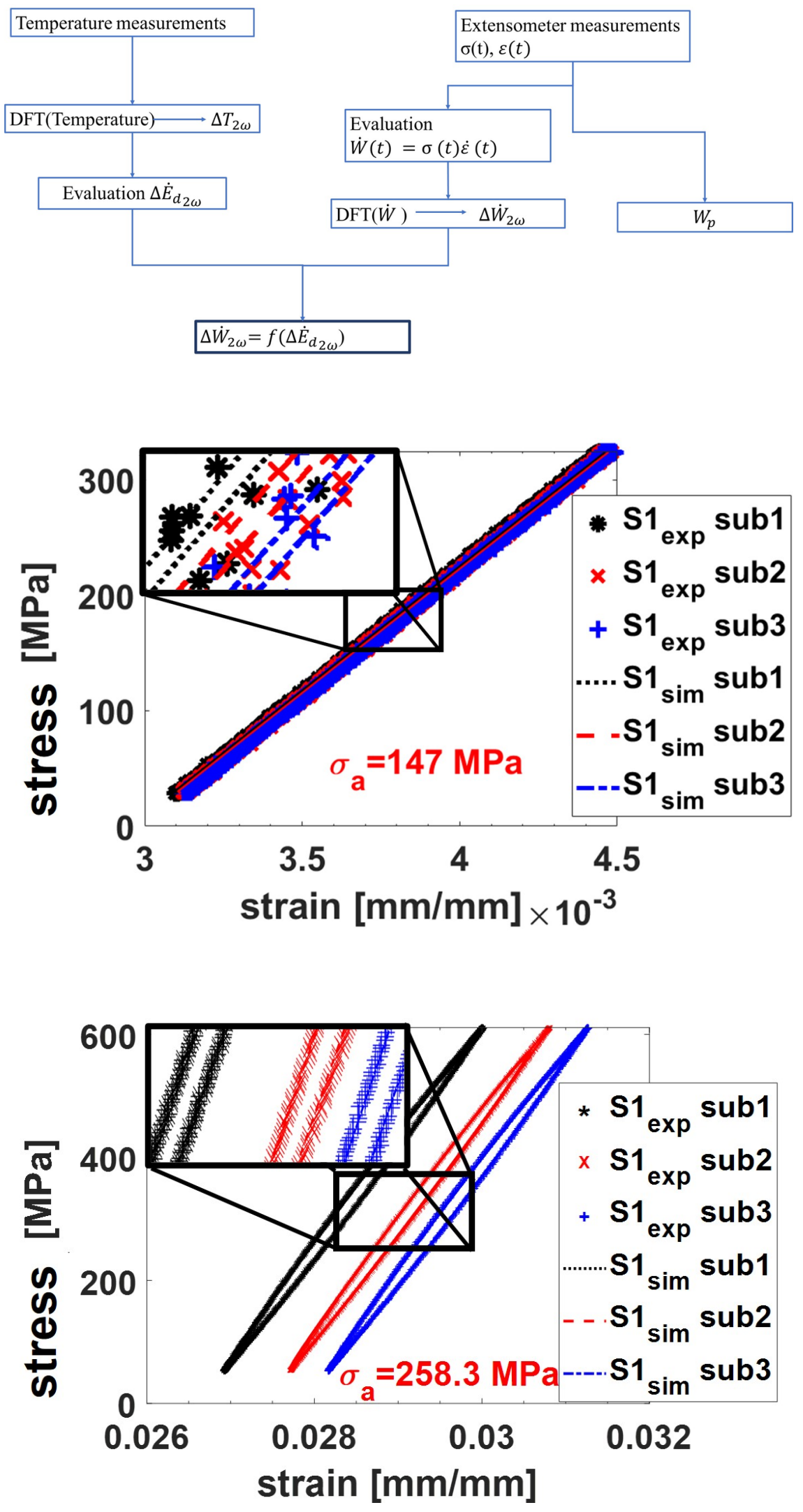

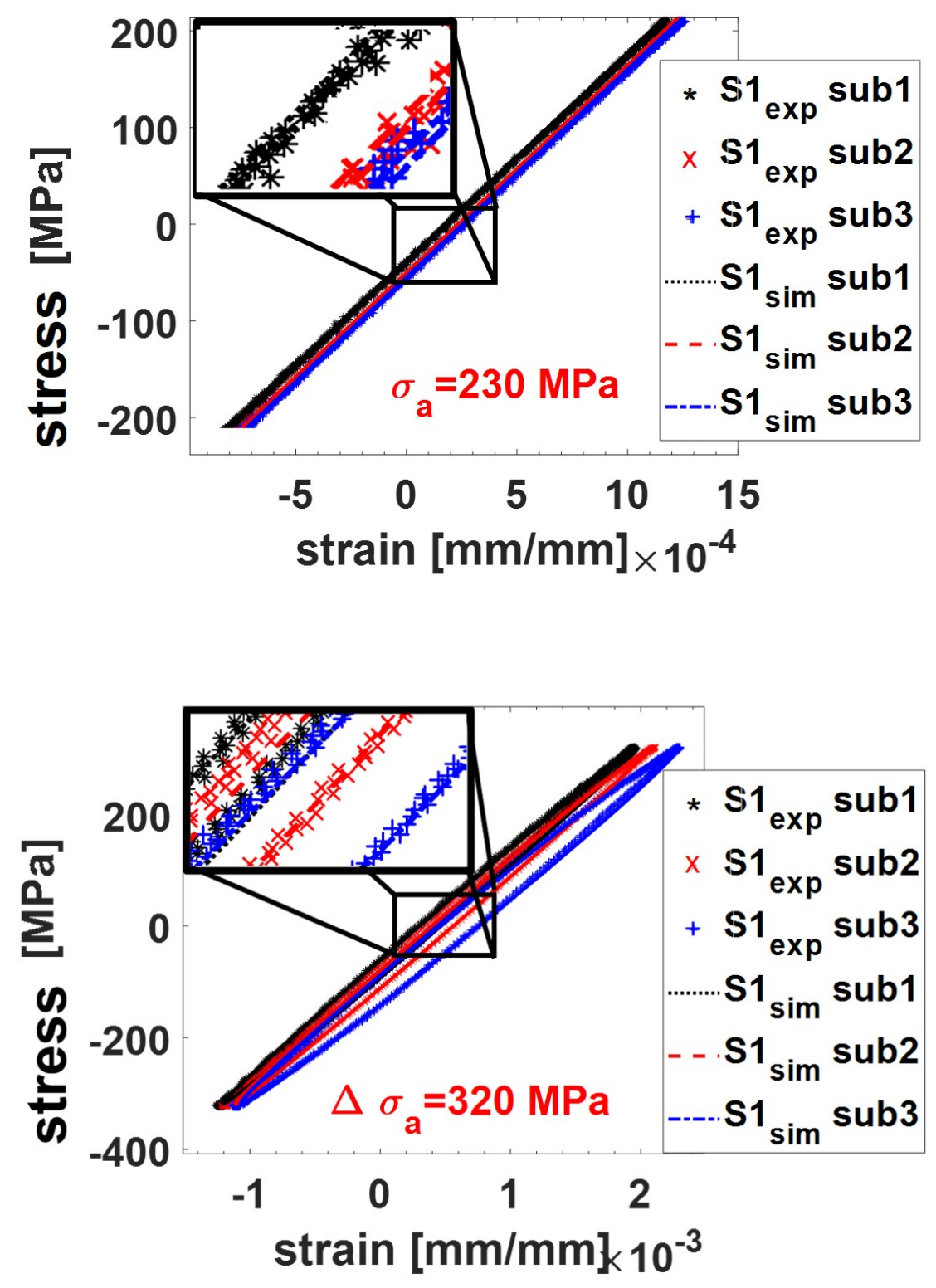

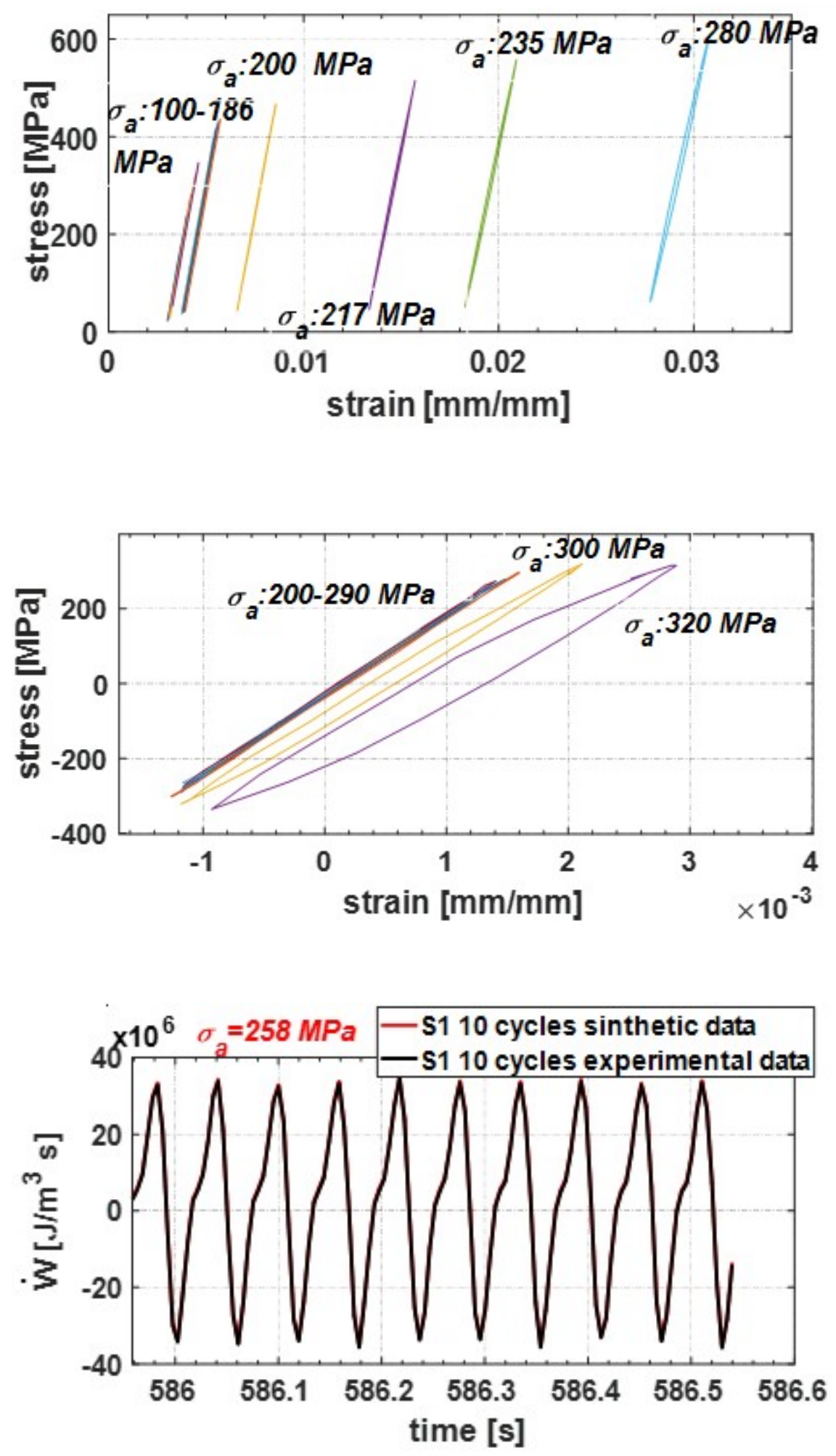

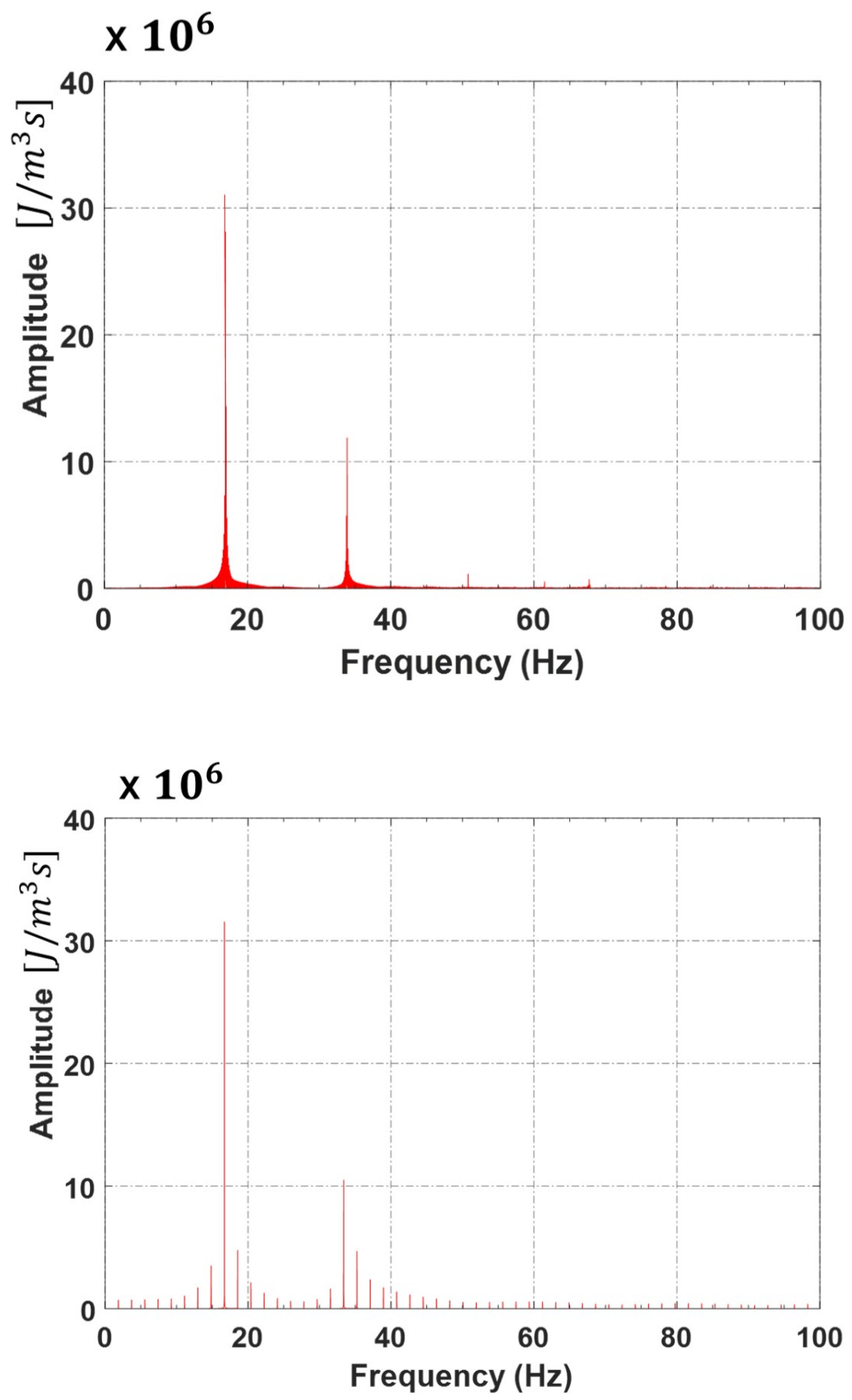

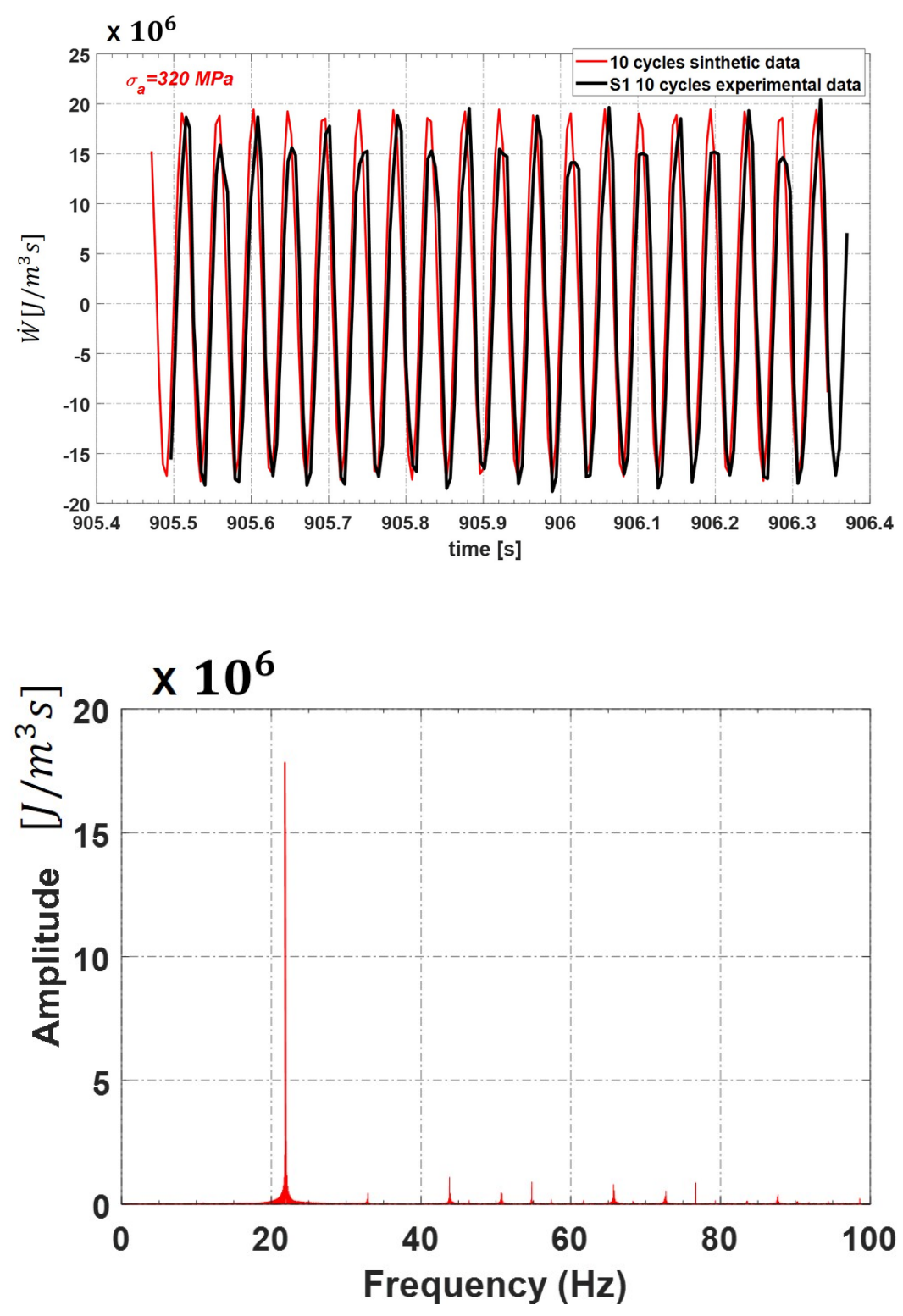

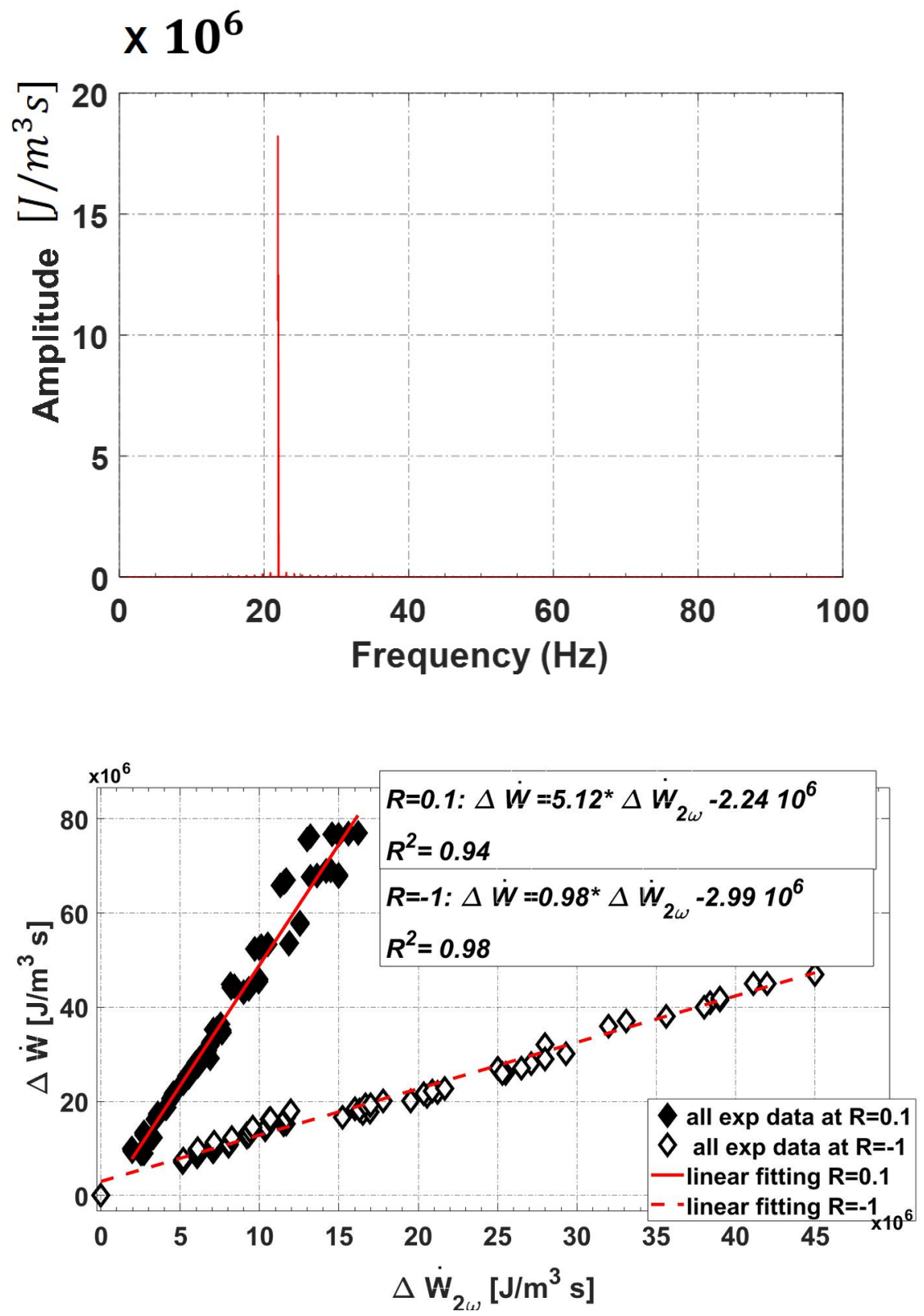

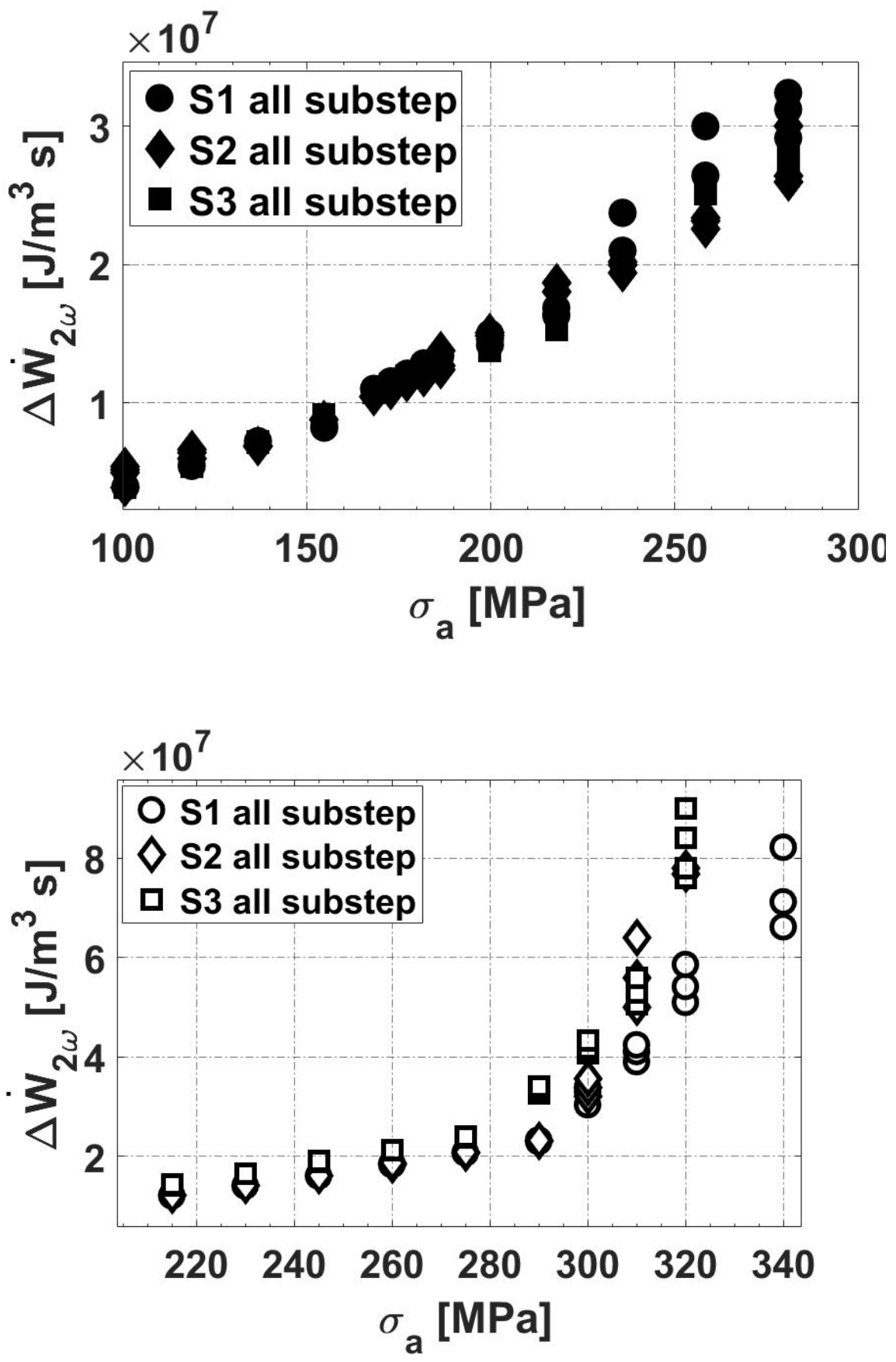

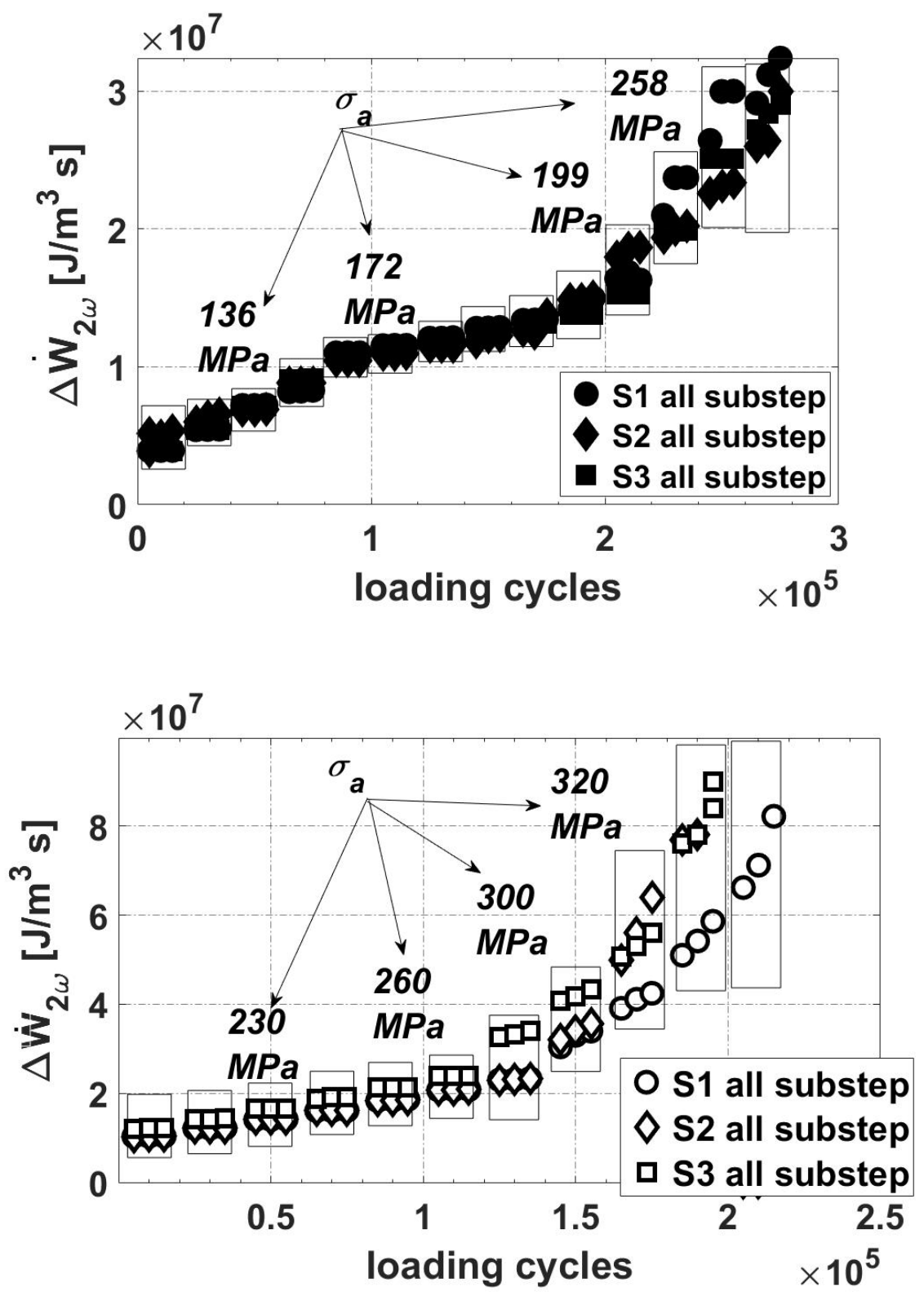

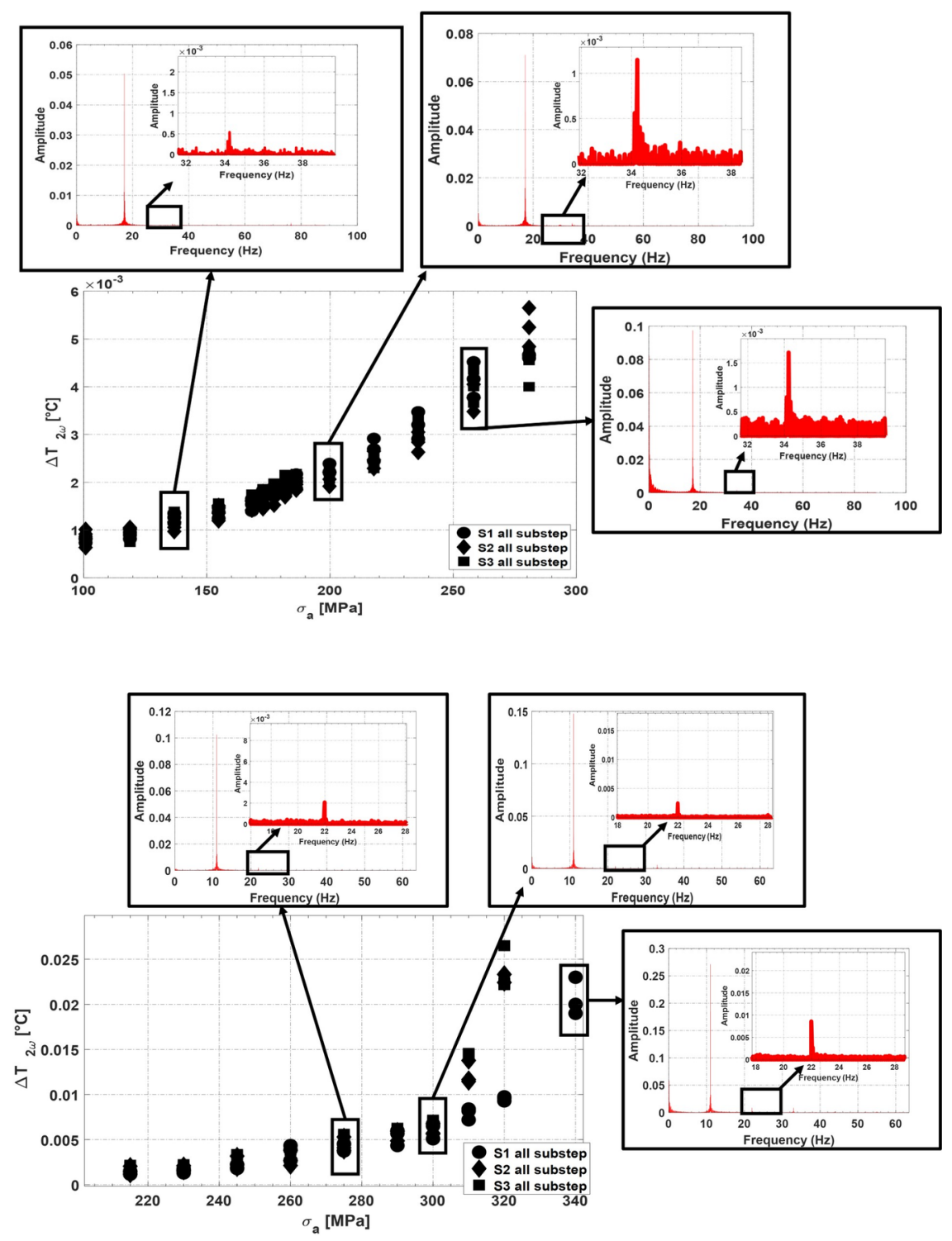

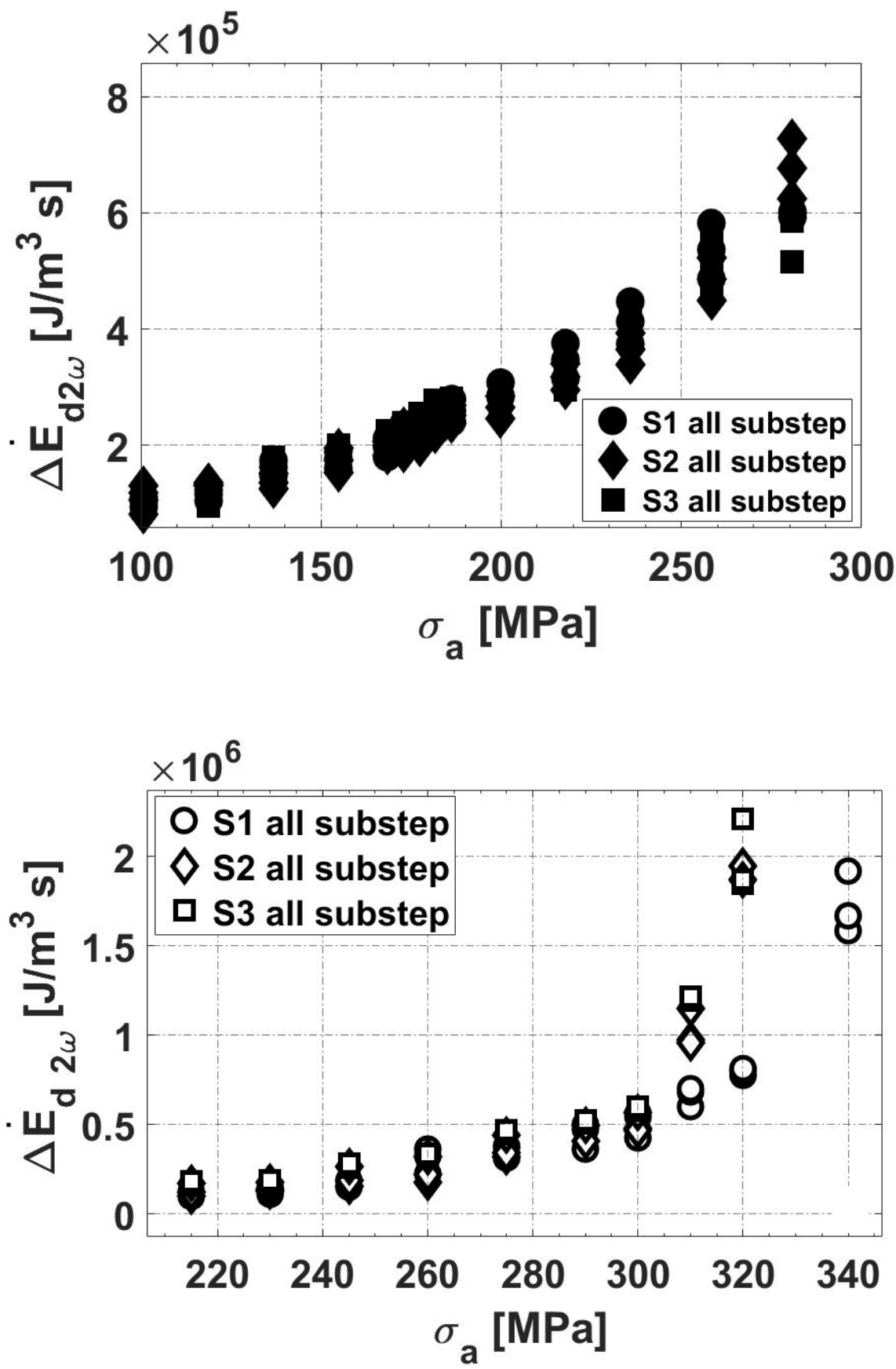

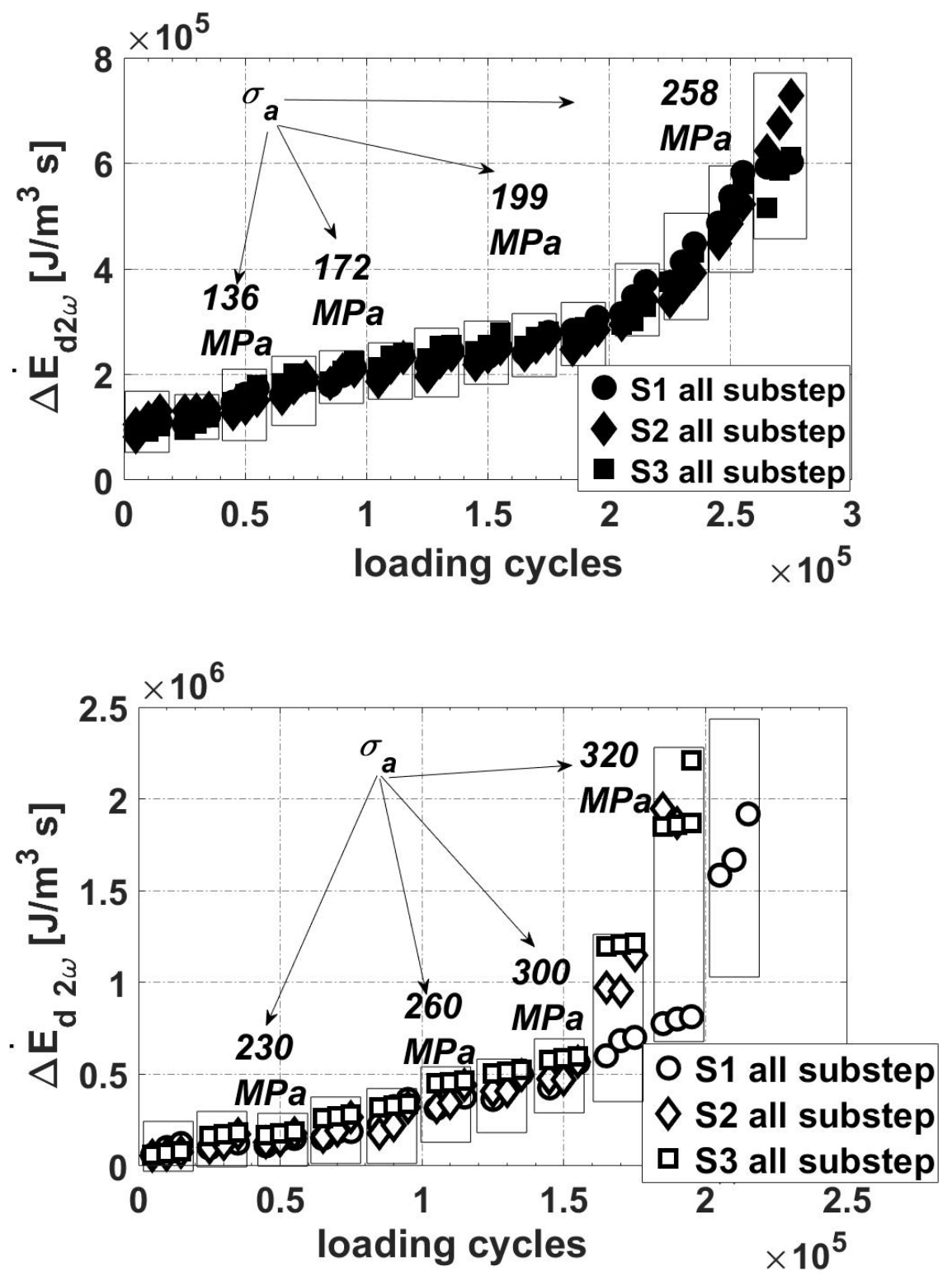

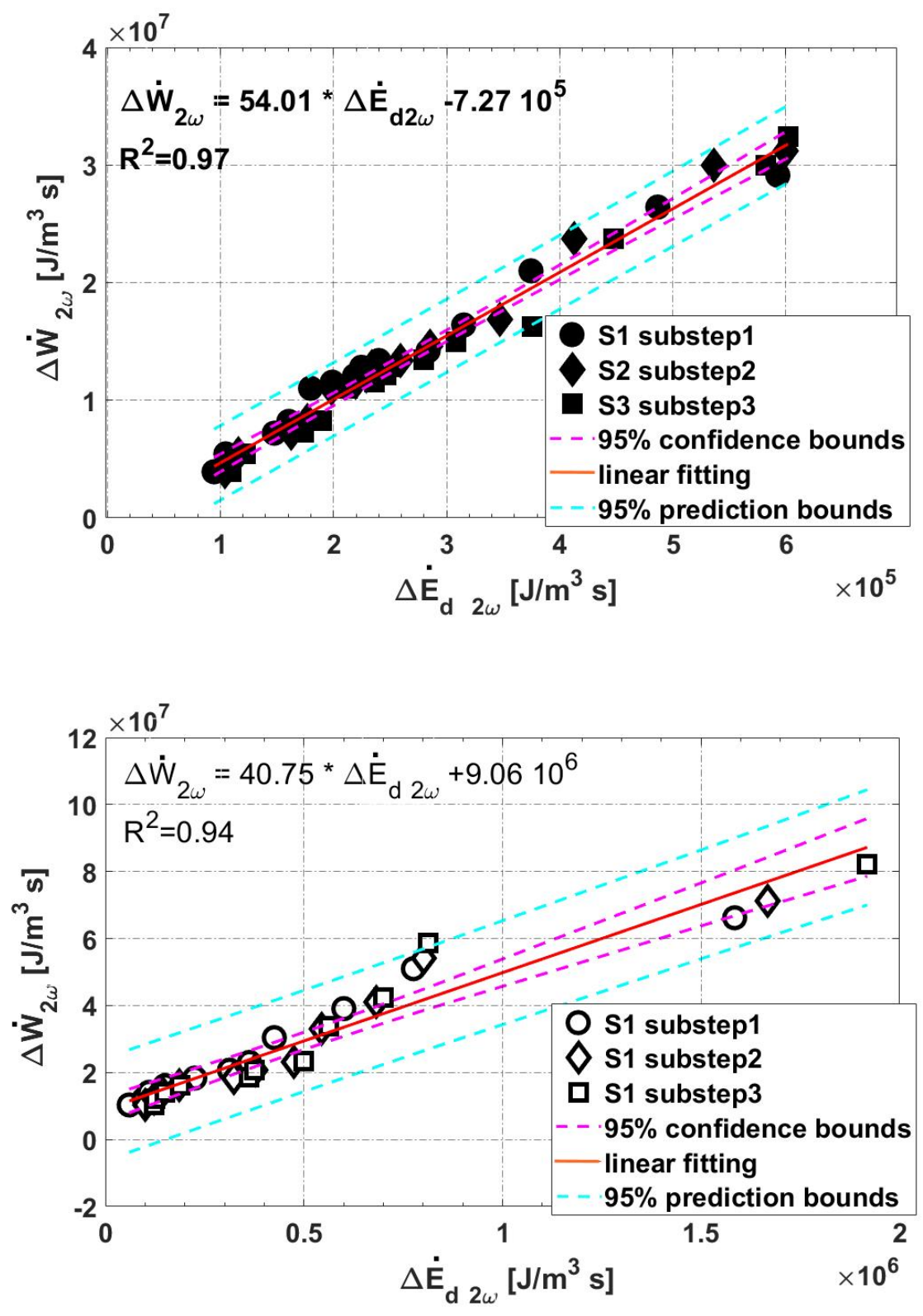

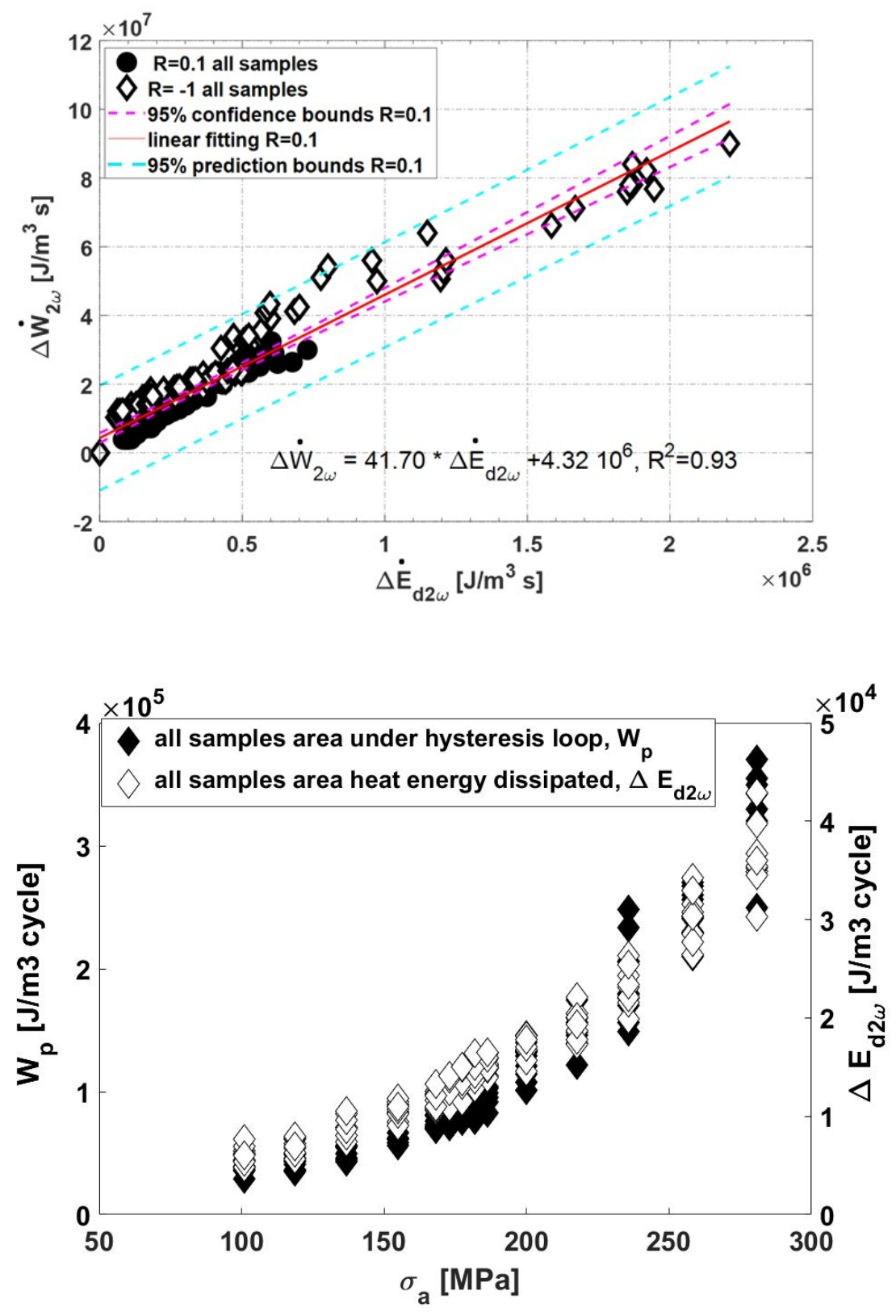


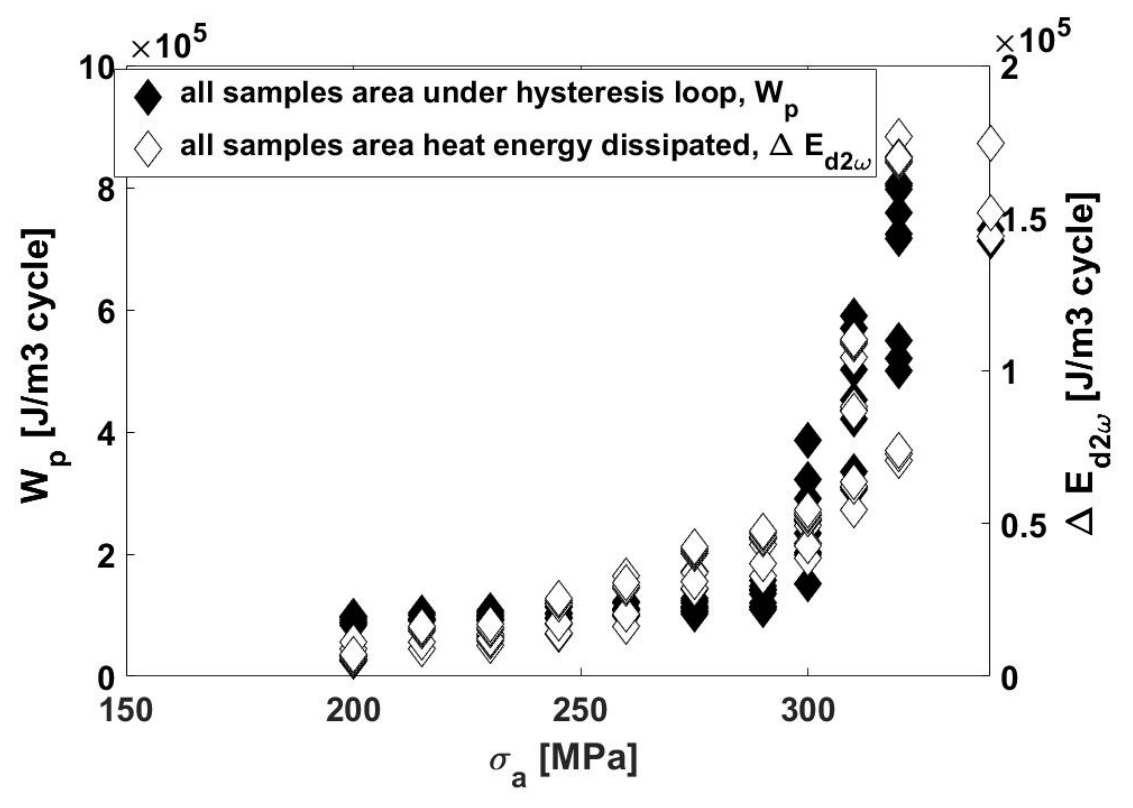

